\title{
Prevalence of COVID-19-related symptoms by age group
}

\author{
Brigid Unim $^{1}\left[\right.$ Luigi Palmieri $^{1} \cdot$ Cinzia Lo Noce ${ }^{1} \cdot$ Silvio Brusaferro ${ }^{2} \cdot$ Graziano Onder $^{1}$
}

Received: 18 January 2021 / Accepted: 2 February 2021 / Published online: 1 March 2021

(C) The Author(s), under exclusive licence to Springer Nature Switzerland AG part of Springer Nature 2021

\begin{abstract}
Background Severe acute respiratory syndrome coronavirus 2 has caused over 95 million confirmed cases of COVID-19 and over 2 million deaths worldwide. According to current literature data, older adults have higher risk of severe disease and mortality due to COVID-19. It is also known that older adults often do not present typical symptoms of diseases. The aim of the study was to assess if the prevalence of typical COVID-19-related symptoms varies by age group.

Methods Medical charts of a random sample of COVID-19 patients dying in-hospital were retrieved through an integrated national surveillance system and reviewed by a group of researchers at the Italian National Institute of Health. Detailed information on COVID-19-related symptoms were extracted and analyzed.

Results 3241 confirmed cases of COVID-19-related deaths were identified from 4391 reviewed medical charts. The mean number of COVID-19-related symptoms progressively declined with age, from 2.1 in patients aged $<60$ years to 1.7 in those aged 90 years or older $(p<0.001)$. Moreover, fever, cough, and diarrhea significantly declined with increasing age.

Conclusions Older adults have atypical presentation of symptoms and may be paucisymptomatic. This may lead to a diagnostic and therapeutic delay which aggravates the prognosis of COVID-19.

Special attention should be posed when assessing individuals aged 65 years and older with suspected COVID-19.
\end{abstract}

Keywords COVID-19 $\cdot$ Older adults $\cdot$ Symptoms $\cdot$ SARS-CoV-2

Current scientific evidence indicates that older adults have higher risk of severe disease and mortality due to COVID19 , particularly those with comorbidities $[1,2]$. The main symptoms of COVID-19, as identified by the Centers of Disease and Control and Prevention, include fever, cough, shortness of breath or difficulty breathing, fatigue, muscle or body aches, headache, loss of taste or smell, sore throat, congestion or runny nose, nausea or vomiting, and diarrhea [3]. However, it is well documented that clinical manifestations of many conditions vary with age and that older adults often do not present typical symptoms of diseases $[4,5]$. This can delay diagnosis and worsen prognosis in this population. The aim of the study was to assess if the prevalence of typical COVID-19-related symptoms varies by age group.

Brigid Unim

brigid.unim@iss.it

1 Department of Cardiovascular, Endocrine-Metabolic Diseases and Aging, Istituto Superiore di Sanità, Via Giano della Bella 34, 00162 Rome, Italy

2 Office of the President, Istituto Superiore di Sanità, Rome, Italy
This analysis was performed in patients dying in-hospital with COVID-19 to define a homogeneous sample and limit possible variability in symptoms presentation due to disease severity.

At the outset of the COVID-19 outbreak, the Italian National Institute of Health (Istituto Superiore di SanitàISS) launched an integrated national surveillance system to collect information on individuals with COVID-19 from all 19 Italian Regions and the two autonomous provinces of Trento and Bolzano [1]. Medical charts of a random sample of 4391 patients dying in-hospital were reviewed by a group of researchers at ISS to obtain more detailed information. To compile the medical charts, all patients were asked about their symptoms with the same standardized procedure through anamnesis. In the presence of critically ill patients, the patients' conditions were reported by relatives, caregivers or health care staff of nursing homes. Data on the following COVID-19-related symptoms prompting hospital admission were collected by the medical staff: fever, dyspnea, cough, diarrhea, and hemoptysis. The recording of the anamnesis was integrated with medical examinations, such as chest imaging through X-ray or computed tomography 


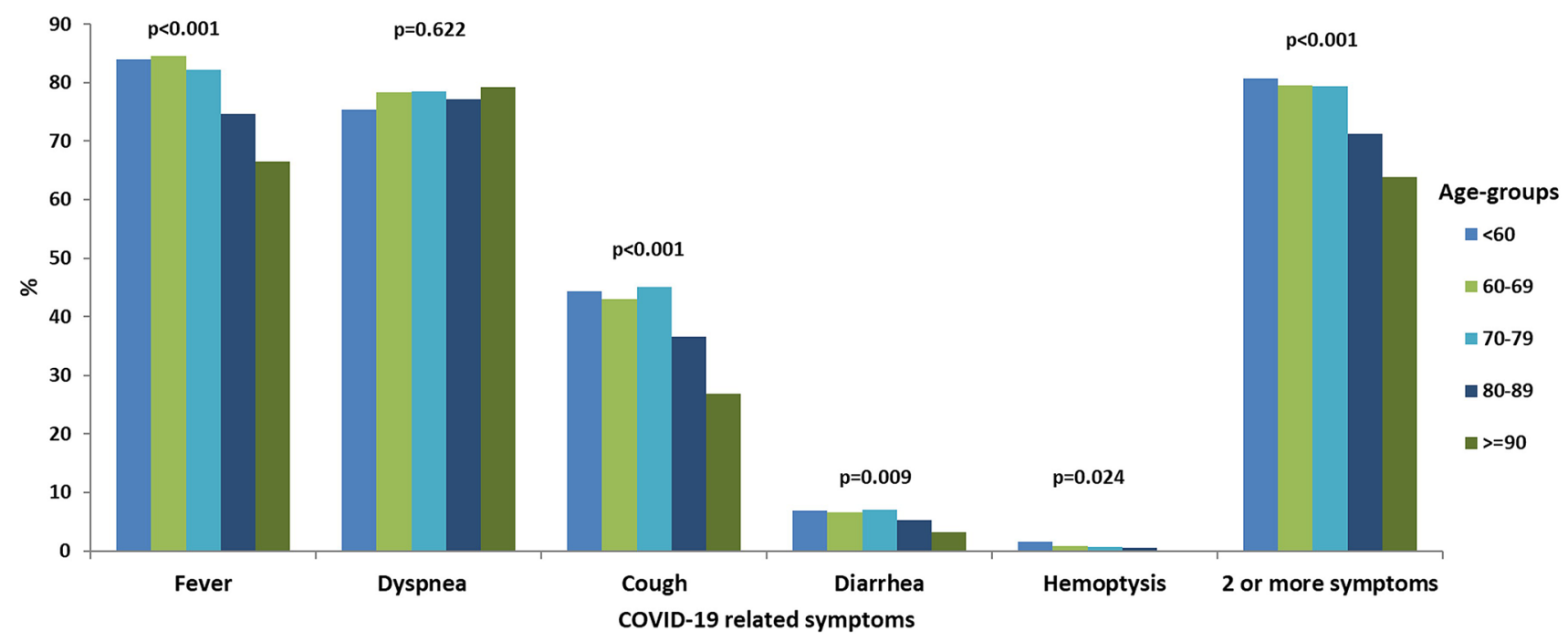

Fig. 1 Prevalence of typical COVID-19-related symptoms by age group

scan, and laboratory assessments through Reverse Transcription Polymerase Chain Reaction (RT-PCR) testing for COVID-19 confirmation. COVID-19-related deaths were defined as those occurring in patients who tested positive for SARS-CoV-2 through RT-PCR test, which is considered the gold standard in SARS-CoV-2 detection. Patients for whom COVID-19 or COVID-19-related conditions (i.e., pneumonia) were not listed in death certificates $(n=543$; $12.4 \%)$, those with an admission diagnosis different from COVID-19 $(n=538 ; 12.3 \%)$ and those with incomplete data on symptoms upon admission due to severe condition limiting their ability to give a full medical history (i.e., patients with mental disorders or intubated) were excluded from the analysis $(n=69 ; 1.6 \%)$. Considering that symptoms were collected from patients with serious COVID-19 disease, early symptoms typical of old age, such as those involving the sensorium, may have been missed.

Differences between age groups were evaluated with chisquare test or Fisher's exact test when appropriate. All analyses were performed using SPSS v. 26.0 (IBM, NY, USA).

Overall, among 3241 cases of COVID-19-related deaths, $74.2 \%$ had two or more symptoms (Fig. 1). Mean number of symptoms progressively declined with age from 2.1 in patients aged $<60$ years, 2.1 in $60-69$, and 2.1 in $70-79$, to 1.9 in those aged $80-89(n=1301)$ and to 1.7 in those aged 90 years or older $(n=40)(p<0.001)$. The difference in mean number of symptoms was also statistically significant between patients aged $<80$ years and those aged 80 years or older ( 2.1 vs $1.9 ; p<0.001)$. Rate of patients with two or more symptoms progressively declined with age from $80.7 \%$ in those aged $<60$ years to $63.8 \%$ in those aged 90 years or older. Presence of fever, cough, diarrhea and hemoptysis progressively and significantly declined with increasing age.
In conclusion, the older adult population have atypical presentation of symptoms and may be paucisymptomatic even in the case of life-threatening health conditions. Age and disease severity may also affect the patient's reporting ability. This may lead to a diagnostic and therapeutic delay which aggravates the prognosis of COVID-19. Particular attention should be posed when evaluating individuals aged 65 years and older with suspected COVID-19.

Acknowledgements We thank the following members of the Italian National Institute of Health COVID-19 Mortality Group who were involved in the collection of data used in this Letter to the Editor: Elvira Agazio, Xanthi Andrianou, Pierfrancesco Barbariol, Antonino Bella, Stefania Bellino, Eva Benelli, Luigi Bertinato, Stefano Boros, Gianfranco Brambilla, Giovanni Calcagnini, Marco Canevelli, Maria Rita Castrucci, Federica Censi, Alessandra Ciervo, Elisa Colaizzo, Fortunato D'Ancona, Martina Del Manso, Corrado Di Benedetto, Chiara Donfrancesco, Massimo Fabiani, Francesco Facchiano, Antonietta Filia, Marco Floridia, Fabio Galati, Marina Giuliano, Tiziana Grisetti, Cecilia Guastadisegni, Yllka Kodra; Martin Langer, Ilaria Lega, Pietro Maiozzi, Fiorella Malchiodi Albedi, Valerio Manno, Margherita Martini, Alberto Mateo Urdiales, Eugenio Mattei, Claudia Meduri, Paola Meli, Giada Minelli, Manuela Nebuloni, Lorenza Nisticò, Marino Nonis, Lucia Palmisano, Nicola Petrosillo, Patrizio Pezzotti, Flavia Pricci, Ornella Punzo, Vincenzo Puro, Federica Quarata, Valeria Raparelli, Simone Rocchetto, Giovanni Rezza, Flavia Riccardo, Maria Cristina Rota, Paolo Salerno, Giulia Sarti, Debora Serra, Andrea Siddu, Paola Stefanelli, Manuela Tamburo De Bella, Dorina Tiple, Marco Toccaceli Blasi, Federica

Trentin, Luana Vaianella, Nicola Vanacore, Maria Fenicia Vescio, Monica Vichi, Emanuele Rocco Villani, Amerigo Zona.

Author contributions Brigid Unim: Conceptualization, Methodology, Investigation, Writing-Original Draft, Writing-Review \& Editing. Luigi Palmieri: Methodology, Data Curation, Formal analysis, Writing-Review \& Editing. Cinzia Lo Noce: Data Curation, Visualization, Writing-Review \& Editing. Silvio Brusaferro: Supervision, 
Writing-Review \& Editing. Graziano Onder: Conceptualization, Methodology, Project administration, Writing-Original Draft, Writing-Review \& Editing. All authors read and approved the final manuscript.

Funding No funding was received for conducting this study.

Availability of data and material The datasets generated during and/or analyzed during the current study are available from the corresponding author on reasonable request.

\section{Compliance with ethical standards}

Conflict of interest No financial relationships with any organization that might have an interest in the submitted work in the previous 3 years; no other relationships or activities that could appear to have influenced the submitted work.

Ethical approval On February 27th, 2020, the Italian Presidency of the Council of Ministers authorized the collection and scientific dissemination of data related to COVID-19 by the Italian National Institute of Health (ISS).

Statement of human and animal rights All procedures performed were in accordance with the ethical standards of the Italian National Institute of Health (ISS).

Informed consent No informed consent was required.

\section{References}

1. Palmieri L, Vanacore N, Donfrancesco C et al (2020) Clinical characteristics of hospitalized individuals dying with COVID-19 by age group in Italy. J Gerontol A Biol Sci Med Sci 75:17961800. https://doi.org/10.1093/gerona/glaa14

2. Palmieri L, Palmer K, Lo Noce C et al (2020) Differences in the clinical characteristics of COVID-19 patients who died in hospital during different phases of the pandemic: national data from Italy. Aging Clin Exp Res 21:1-7. https://doi.org/10.1007/s40520-02001764-0

3. Centers of Disease and Control and Prevention (2020) Coronavirus Disease 2019 (COVID-19). https://www.cdc.gov/coronaviru s/2019-ncov/symptoms-testing/symptoms.html. Accessed 23 December 2020

4. Wester AL, Dunlop O, Melby KK, Dahle UR, Wyller TB (2013) Age-related differences in symptoms, diagnosis and prognosis of bacteremia. BMC Infect Dis 13:346. https://doi. org/10.1186/1471-2334-13-346

5. Nanda A, Vura NVRK, Gravenstein S (2020) COVID-19 in older adults. Aging Clin Exp Res 32:1199-1202. https://doi. org/10.1007/s40520-020-01581-5

Publisher's Note Springer Nature remains neutral with regard to jurisdictional claims in published maps and institutional affiliations. 